Revue d'histoire de l'Amérique française

REVUE D.HISTOIRE DE L'AMÉRIQUE FRANÇAISE

\title{
La justice seigneuriale en Nouvelle-France : le cas de Notre-Dame-des-Anges
}

John A. Dickinson

Volume 28, numéro 3, décembre 1974

URI : https://id.erudit.org/iderudit/303366ar

DOI : https://doi.org/10.7202/303366ar

Aller au sommaire du numéro

Éditeur(s)

Institut d'histoire de l'Amérique française

ISSN

0035-2357 (imprimé)

1492-1383 (numérique)

Découvrir la revue

Citer cet article

Dickinson, J. A. (1974). La justice seigneuriale en Nouvelle-France : le cas de Notre-Dame-des-Anges. Revue d'histoire de l'Amérique française, 28(3), 323-346. https://doi.org/10.7202/303366ar d'utilisation que vous pouvez consulter en ligne.

https://apropos.erudit.org/fr/usagers/politique-dutilisation/ 


\title{
LA JUSTICE SEIGNEURIALE EN NOUVELLE-FRANCE: LE CAS DE NOTRE-DAME-DES-ANGES
}

\author{
John A. Dickinson \\ Université de Toronto
}

Les structures administratives en Nouvelle-France ont peu retenu l'attention des historiens jusqu'à présent. Gustave Lanctot, Jean Delalande et Raymond Du Bois Cahall ${ }^{1}$ ont assez bien décrit les cadres supérieurs de l'administration coloniale et le Conseil souverain. André Vachon a énuméré rapidement les divers appareils administratifs internes, mais sans analyser leur fonctionnement ${ }^{2}$. Cependant des institutions subalternes telles que les bureaux de la Marine, le Domaine d'Occident, la maréchaussée et surtout les cours de justice seigneuriale jouèrent un rôle important dans la vie coloniale et mériteraient d'être mieux connues.

La plupart des auteurs de synthèses ne se sont guère intéressés aux juridictions seigneuriales; tantôt les qualifiant de négligeables ${ }^{3}$, tantôt affirmant que quelques seigneurs seulement exerçaient leurs droits ${ }^{4}$. Certains, comme Marcel Trudel, soutiennent que la plupart des seigneurs avaient droit de haute justice mais qu'en pratique, après 1693, leurs compétences furent

${ }^{1}$ Gustave Lanctot, L'administration de la Nouvelle-France (Paris, Librairie Honoré Champion, 1929) ; Jean Delalande, Le Conseil souverain de la Nouvelle-France (Québec, Ls.-A. Proulx, Imprimeur du Roi, 1927); Raymond Du Bois Cahall, The Sovereign Council of New France (New York, Longmans, Green \& Co., 1915).

2 André Vachon, "L'administration de la Nouvelle-France", in Dictionnaire biographique du Canada (Québec, Presses de l'Université Laval, 1970), II : xv-xxiv.

3 Parkman affirme que leur compétence ne s'étendait qu'aux causes triviales. Francis Parkman, The Old Régime in Canada (Boston, Little, Brown \& Co., 1898), 302. 'Diamond assure qu'elles ne pouvaient concurrencer les justices royales et que leur juridiction se limita aux causes concernant les cens et rentes seigneuriales. Sigmund Diamond, "Old patterns and new societies: Virginia and New France in the seventeenth century", in Werner J. Cahnman and Alvin Boskoff, Sociology and history (New York, The Free Press, 1964), 177.

4 William John Eccles, France in America (Toronto, Fitzhenry \& Whiteside Limited, 1973), 73.

RHAF, vol. 28, no 3 (décembre 1974) 
réduites à la moyenne et à la basse justice ${ }^{5}$. L'on n'en traite guère davantage dans les monographies relatives au régime seigneurial. Trudel n'offre rien de neuf à ce sujet ${ }^{6}$. D'après E. R. Adair, les seigneurs n'avaient jamais exercé leur droit de haute justice et seule la basse justice aurait eu un effet quelconque ${ }^{7}$. Richard Colebrook Harris, dans une des meilleures études sur le régime seigneurial, se débarrasse de la justice en moins d'une page, reprenant les affirmations d'Adair ${ }^{8}$. William Bennett Munro, qui traite le plus longuement de ce problème, s'approche de la réalité quand il avance que la majorité des seigneurs avaient droit de haute justice et que certaines cours furent bien établies et fonctionnèrent normalement ${ }^{9}$. Aucun de ces auteurs ne révèle le caractère exact de ce niveau judiciaire.

La confusion qui règne autour de l'étude des justices seigneuriales serait, en grande partie, imputable au manque de documents, comme l'ont affirmé Joseph-Edmond Roy et W. B. Munro ${ }^{10}$. Il en existe cependant quelques petites collections ${ }^{11}$ et au moins une série assez considérable: celle de la justice de Notre-Dame-des-Anges. Les registres de cette juridiction sont

5 Marcel Trudel, Initiation à la Nouvelle-France (Montréal et Toronto, Holt, Rinehart \& Winston, 1968), 228-229. André Vachon est aussi de cet avis, André Vachon, loc. cit.: xxiii.

6 Marcel Trudel, The Seigneurial Regime (Ottawa, Canadian Historical Association, 1967), booklet, no 6:11.

7 E. R. Adair, "The French-Canadian Seigneury", Canadian Historical Review (1954), XXXV: 196.

8 Richard Colebrook Harris, The Seigneurial System in Early Canada (Québec, Les Presses de l’Université Laval, 1966), 190-191.

9 William Bennett Munro, The Seigniorial System in Canada (New York, Longmans, Green \& Co., 1907), 151.

10 J.-Edmond Roy, La justice seigneuriale de Notre-Dame-des-Anges (s.l.n.d.), 5, et Munro, op. cit., 155.

11 Lucille Labrèque, "Inventaire de pièces détachées de cours de justice de la Nouvelle-France (1638-1760)", Rapport des Archives nationales du Québec, 49 (1971) : 5-9 et 24-40. Dans l'introduction de cet inventaire le Conservateur en chef des Archives affirme erronément que: "Seuls quelques fragments et quelques pièces éparses ont été conservés, qui ont du fait même de leur rareté une valeur inestimable. Nous présentons ici l'inventaire de ces débris que nous conservons aux Archives nationales du Québec" (p. 3). Il est étonnant que le Conservateur semble ignorer l'existence d'un fonds aussi important que celui de Notre-Dame-des-Anges. Plus grave encore, une telle affirmation de la part d'une autorité compétente contribue à perpétuer l'idée que ces cours ne peuvent être analysées à défaut de documents. 
conservés aux Archives nationales du Québec dans la Collection de pièces judiciaires et notariales ${ }^{12}$.

Bien que ce fonds constitue une documentation très riche, son analyse comporte plusieurs problèmes sérieux. A défaut d'inventaire, il fallut dépouiller minutieusement tous les registres et actes de cette série. Ceci nous permit de remédier au grand désordre dans la classification des documents (dates qui ne se suivaient pas, présence de registres de Beauport, de Beaupré et même de Batiscan!) et de constater avec précision la nature et le contenu des pièces encore existantes de cette juridiction. Les lacunes sont malheureusement nombreuses: plusieurs registres manquent complètement tandis que d'autres ne sont que partiels. A titre d'exemple: aucune pièce provenant des années 1681, 1682, 1690, 1691 et 1703 ne s'y trouve et seulement un document pour les années 1680,1683 et 1709 subsiste. Souvent les procès sont incomplets: soit les étapes préliminaires, soit le jugement définitif manquent; en conséquence plusieurs détails concernant certaines causes demeurent inconnus. Les frais et émoluments sont rarement indiqués et lorsqu'ils le sont, ils ne donnent tantôt en "livres de france", tantôt en "monnaye du pays", que le coût total du procès sans énumération de la part attribuable à chaque officier. Ainsi la documentation recueillie sur ce plan nous laisse sur notre appétit.

Il est difficile d'expliquer les lacunes. Joseph-Edmond Roy fournit un indice qui pourrait éclairer ce problème: un conflit entre les juridictions de Notre-Dame-des-Anges et de Beauport au sujet des archives du greffe. Paul Vachon, Jean Robert et Noël Duprac avaient successivement cumulé les fonctions de greffier dans les deux juridictions pendant près d'un siècle. Au décès de Noël Duprac en 1748, tous les documents en sa possession furent déposés ensemble au greffe de Beauport. Ce ne fut qu'en 1750 que le juge de Notre-Dame-des-Anges, Paul-Antoine-François Lanoullier, s'occupa de la récupération des registres. Au cours du procès qui dura un an, il paraît qu'Antoine Juchereau Duchesnay, seigneur de Beauport, "les fit enlever furtivement, et mêler ensemble confusément". Les archives furent finalement

12 Un inventaire existe, celui de Pierre-Georges Roy, mais il est trop incomplet et contient de nombreuses erreurs surtout en ce qui concerne les dates. Le répertoire des actes est assez complet, mais quand il s'agit des registres d'audience il ne met que: "registre pour l'année..." sans élaborer davantage. Pierre-Georges Roy, Inventaire d'une collection de pièces judiciaires et notariales conservées aux Archives judiciaires de Québec (Beauceville, L'Eclaireur, 1917), I: 255-280. 
remises au greffier de Notre-Dame-des-Anges en $1755^{13}$, mais on ne se serait guère préoccupé de démêler et de vérifier systématiquement le contenu de tous les registres, ce qui expliquerait certaines lacunes et le désordre.

Malgré ces déficiences, la série de Notre-Dame-des-Anges se révèle assez complète pour permettre une analyse des compétences et $\mathrm{du}$ fonctionnement d'une justice seigneuriale. L'examen de la nature des causes jugées, du déroulement des séances, du nombre de procès-verbaux et d'actes dressés par la cour précise le rôle de ces cours subalternes. A partir des informations relatives aux dépens des procès se dégage un aperçu des frais qu'encouraient les plaideurs à ce niveau judiciaire. Enfin nous essayerons de déterminer quels avantages le seigneur pouvait retirer de l'établissement d'une justice dans sa seigneurie.

\section{La juridiction seigneuriale de Notre-Dame-des-Anges}

La juridiction seigneuriale, c'est le pouvoir de juger qui est octroyé à un individu ou à un organisme, ainsi que l'aire géographique sur laquelle ce droit peut être exercé. Elle peut englober une seule seigneurie, ou être étendue à plusieurs, comme ce fut le cas de Notre-Dame-des-Anges. Au cœur de ce district se trouvait le fief de Notre-Dame-des-Anges, le premier concédé aux Jésuites au Canada en 1626. D'une superficie d'environ cinq lieues, il longeait le Saint-Laurent et la rivière SaintCharles, du ruisseau de Beauport, à l'est, au ruisseau SaintMichel, à l'ouest ${ }^{14}$. Une cour y siégea à compter d'au moins $1664{ }^{15}$. En vertu d'un accord entre les seigneurs des petits fiefs de Saint-Ignace, Lépinay et Orsainville, la même personne exerçait ses fonctions dans toutes les seigneuries et toutes les séances se tenaient dans la salle d'audience de Notre-Dame-desAnges ${ }^{16}$. Au fur et à mesure de la concession ou de l'achat des

13 J.-Edmond Roy, op. cit., 15-23.

14 Ibid., 2-3.

15 Archives nationales de Québec, Biens des Jésuites, cote QBC 20, le $1 \mathrm{er}$ août 1664 . Nous retrouvons à cette date la commission de Claude Bermen de la Martinière pour exercer les fonctions de juge à Notre-Damedes-Anges. La série dans la Collection de pièces judiciaires et notariales ne débute qu'en 1679 .

16 J.-Edmond Roy, op. cit., 7. Roy ne spécifie pas la date de cette entente. Duquet reçut deux commissions, l'une comme juge de Notre-Damedes-Anges en 1679 et l'autre pour Orsainville en 1680. J.-Edmond Roy, Histoire du notariat au Canada (Lévis, La Revue du Notariat, 1889), 90. Deux commissions existent aussi pour Lanoullier, une pour Notre-Dame- 
trois seigneuries de Sillery, Saint-Gabriel et Bélair, les Jésuites étendirent la compétence de la cour de Notre-Dame-des-Anges pour les inclure dans la juridiction. Les seigneurs eurent beaucoup de mal à établir leur droit de justice à Sillery, surtout à cause des remontrances du procureur général, Ruette d'Auteuil ${ }^{17}$. En 1704 leur cause semblait perdue. Le roi, dans un mémoire au gouverneur et à l'intendant, manifesta son opposition à l'établissement d'une justice à Sillery ${ }^{18}$. Cependant les administrateurs coloniaux semblent avoir ignoré cette dépêche puisqu'un procureur fiscal fut reçu par le juge Haimard le 17 décembre de la même année ${ }^{19}$. Le dénouement de cette histoire eut lieu en 1707 quand l'intendant supprima le droit de haute justice à Sillery; la moyenne et la basse justice furent toutefois maintenues ${ }^{20}$. A l'exemple d'un grand nombre de communautés religieuses françaises, les Jésuites regroupèrent toutes leurs terres de la région de Québec dans une même juridiction afin de rendre la justice plus rentable ${ }^{21}$. La juridiction de NotreDame-des-Anges s'étendit à la majeure partie des terres autour de la capitale coloniale, de Beauport à Cap-Rouge sur quelque 260.000 arpents ${ }^{22}$.

La population de la juridiction était assez importante. En 1681, elle comptait environ 750 âmes, soit, à peu près 140

des-Anges et l'autre pour Lépinay. Archives nationales du Québec, Collection de pièces judiciaires et notariales, (ci-après $C P J N$ ), folio 3012 , le 12 mars 1750 et Archives nationales du Québec, Registres de la Prévôté de Québec (vol. 98) le 5 mai 1750.

17 François-Madeleine-Fortuné Ruette d'Auteuil, "Lettres et mémoires de François-Madeleine-Fortuné Ruette d'Auteuil, procureur général du Conseil souverain de la Nouvelle-France", Rapport de l'Archiviste de la Province de Québec (1922-1923) : 30.

18 Pierre-Georges Roy, "Correspondance entre Vaudreuil et la cour", Rapport de l'Archiviste de la Province de Québec (1938-1939), Mémoiré du Roi à MM. de Vaudreuil et de Beauharnois du 14 juin 1704, 42.

$19 C P J N$, folio 2794, le 17 décembre 1704: installation d'André Maufait dans ses fonctions de procureur fiscal de Sillery.

20 Province de Québec, Edits, ordonnances royaux, déclarations et arrêts du Conseil d'Etat du Roi concernant le Canada (Imprimé sur une adresse de l'Assemblée législative du Canada, Québec, F.-R. Fréchette, 1854), III : 138.

21 Amédée Combier, Les justices seigneuriales du bailliage de Vermandois sous l'ancien régime (Paris, Fontemoing, 1897), 14. Apparemment cette pratique ne fut pas restreinte aux Jésuites en Nouvelle-France puisque nous trouvons des commissions pour la Grande Anse et la Bouteillerie $(C P J N$, folio 2018) et Yamaska et Saint-François (CPJN, folios $20731 / 2$ et 2076 ).

22 Munro, op. cit., 180. Une carte de $1688 \mathrm{du}$ Sieur de Villeneuve, conservée aux Archives nationales du Québec (cote: AQ, B 932, Québec 1688) démontre clairement l'étendue de cette juridiction. 
familles ${ }^{23}$. Le recensement de 1706 donnait 869 âmes pour Notre-Dame-des-Anges et Sillery, 142 pour Saint-Ignace et Orsainville, ce qui faisait un total de 1.011 personnes ${ }^{24}$. Lors de la tournée des paroisses effectuée par le procureur général Collet en 1721, on dénombrait environ 250 familles ou près de 1.300 personnes à Sainte-Foy, Lorette et Charlesbourg ${ }^{25}$. La population atteignait 1.700 âmes en $1739{ }^{26}$ et, à la fin du régime français, 340 familles, comprenant en tout 2.845 personnes, habitaient la juridiction ${ }^{27}$. La bonne administration des Jésuites, la proximité de Québec, lieu de débarquement des colons au XVIIe siècle et port d'exportation des surnlus agricoles au XVIIIe siècle, expliquent que cette région ait été une des plus populeuses de la colonie.

Il est difficile de déterminer le niveau de vie de cette population. Harris avance quelques chiffres relatifs à l'exploitation $\mathrm{du}$ sol et au niveau de vie pour Notre-Dame-des-Anges ${ }^{28}$, mais il faut savoir que ces données ne tiennent pas compte du facteur urbain. En effet, plusieurs personnes qui demeuraient sur les rives de la rivière Saint-Charles et sur la côte Saint-Jean étaient des gens de métier occupant de petits emplacements. En outre, des citadins, comme Jacques Pinguet de Vaucour ${ }^{29}$, possédaient, dans les seigneuries de la banlieue, des terres qu'ils affermissaient ou utilisaient pour le pâturage de leur bétail. La majorité des gens poursuivaient toutefois des occupations agricoles et la présence du marché urbain et surtout les possibilités d'exportation des denrées vers Louisbourg et les Iles du Vent devaient assurer un niveau de vie relativement élevé.

23 Canada, Recensement du Canada, 1871 (Ottawa, Imprimeur de la Reine, 1876), IV: 11.

24 Ibid.: 48.

25 La paroisse de Charlesbourg avec ses 14 "villages" et deux "terrains" de Grospin et Saint-Jérôme était la plus considérable avec ses 130 chefs de famille. La paroisse de Lorette comprenait aussi certains censitaires des fiefs de Gaudarville et De Maure, mais à l'est, celle de Beauport réclamait les 17 familles habitant le Petit Village, fait que ces habitants n'appréciaient guère. Invanhoë Caron, "Procès-verbaux du procureur général Collet sur le district des paroisses de la Nouvelle-France", Rapport de l'Archiviste de la Province de Québec (1921-1922): 265-267 et 358-360.

26 Canada, op. cit.: 60.

27 Ibid.: 64.

28 Harris, op. cit., 146-148, 153 et 191.

29 John Dickinson, Les officiers de la justice seigneuriale de NotreDame-des-Anges (1664-1759). Thèse manuscrite de Maîtrise ès Arts (Histoire) (Québec, Université Laval, 1972), 77-78. 


\section{La compétence de la cour seigneuriale}

D'après la coutume française il existait trois niveaux de justice exercés dans les seigneuries: la haute, la moyenne et la basse justice. La haute justice conférait le droit de juger tous les crimes et de prononcer toutes les peines au criminel, à l'exception des cas royaux. Cependant la peine de mort nécessitait la confirmation d'une cour royale. La moyenne justice avait compétence en matière pénale pour les peines allant jusqu'à soixante sols, et, en matière civile, cette compétence s'étendait à toutes les obligations féodales, aux nominations de tuteurs pour les enfants mineurs, aux appositions de scellés lors des décès; elle permettait au juge de procéder à l'inventaire des biens après décès et à la vente aux enchères. La basse justice n'attribuait que le seul pouvoir de trancher des litiges civils portant sur des affaires impliquant moins de soixante sols ${ }^{30}$. Celle-ci ne procurait qu'un prestige et des avantages financiers négligeables au seigneur et aux officiers; la moyenne justice était, sur ces points, plus rentable et accordait au seigneur un mécanisme additionnel pour administrer sa seigneurie. La haute justice avait de la valeur par le prestige qu'elle conférait au seigneur et aux officiers.

Comme la plupart des seigneurs dont les concessions étaient antérieures à 1693, les Jésuites avaient le droit de haute justice à Notre-Dame-des-Anges et cette prérogative fut exercée. Un des exemples les plus significatifs fut l'affaire de Jean Denis. Trouvé coupable de meurtre le 14 septembre 1695 par le juge Guillaume Roger, il fut condamné à mort par pendaison ${ }^{31}$. Un autre procès concernant la découverte du cadavre de Jean Normand entraîna un conflit de juridictions entre la justice seigneuriale et la Prévôté de Québec. Le Conseil supérieur régla le litige par une ordonnance du 2 août 1706 , dans laquelle il affirmait la compétence du juge seigneurial:

Le Sr Lieutenant général en la Prevosté de cette ville et les officiers d'icelle Incompétans de Laffaire dont il sagist, Leur faire defences d'en connoistre et passer outre sous telle peine que de raison et ordonner que les procédures faites en ladite Prevosté seroient apportées en ce Conseil pour estre déclarées

30 Combier, op. cit., 5.

31 Anonyme, "La haute justice dans les justices seigneuriales", Bulletin des Recherches historiques (1917), XXIII: 187-190. Cette sentence ne se trouve pas dans les registres manuscrits, la période entre août 1695 et janvier 1696 étant absente. 
nulles, et que les Informations et autres procédures seroient faites et Continués en la juridiction de ladite Seigneurie. Le Cadavre ayant esté trouve sur Icelle et en dépendant [...] le Conseil [...] ordonne que le juge de la dite Seigneurie de notre Dame des Anges Continuera le procez ainsi qu'il a commencé 32 .

Cette ordonnance ne laisse subsister aucun doute: la haute justice relevait bien des compétences seigneuriales. Elle montre en outre que les justices subalternes ont su se défendre contre les empiétements des instances supérieures.

Ce droit de haute justice était jalousement maintenu moins pour les avantages pécuniaires qui pouvaient en découler que pour le prestige qu'on y attachait. Les instructions criminelles exigeaient beaucoup plus de travail de la part des officiers qu'un procès civil et ce à un tarif bien inférieur ${ }^{33}$. En plus le criminel devait être gardé dans la prison seigneuriale aux frais du seigneur. Ces inconvénients faisaient en sorte qu'en France la plupart des justices seigneuriales négligeaient ces procédures ${ }^{34}$. En Nouvelle-France la même situation devait exister puisque les mêmes règlements régissaient la conduite des procès. A NotreDame-des-Anges les instances criminelles ne furent pas fréquentes. Une autre raison susceptible d'expliquer ce fait est l'ordonnance de 1670. Aux termes de cette ordonnance, tout procès criminel exigeait la présence de trois juges gradués ${ }^{35}$. Le Conseil supérieur, qui estimait que cette règle n'était pas respectée, rappela à l'ordre les justices subalternes en $1714^{36}$. Cette action contribua probablement à persuader les juges seigneuriaux d'abandonner les causes criminelles au profit de la justice royale.

32 CPJN, folio 2800 , le 2 août 1706 .

33 Québec, Edits et ordonnances, I: 99-102.

34 André Giffard, Les justices seigneuriales en Bretagne au XVIIe et XVIIIe siècles, Thèse pour le doctorat, faculté de droit, Université de Paris (Paris, Arthur Rousseau, 1902), 124, et Fernand Braudel, Ernest Labrousse et al, Histoire économique et sociale de la France (Paris, Presses universitaires de France, 1970) II : 123.

35 Le terme gradué implique une formation universitaire. Marcel Marion, Dictionnaire des institutions de la France aux XVIIe et XVIIIe siècles (Paris, A. \& J. Picard, 1968), 263. En Nouvelle-France les gens de cette qualité étaient rares et si le règlement avait été rigoureusement appliqué même la Prévôté aurait eu des problèmes à trouver des juges. Pour contourner cette difficulté on attribuait aux notaires royaux la compétence de siéger dans les affaires criminelles.

36 CPJN, folio 2833. Plainte du procureur général "insinué" à NotreDame-des-Anges le 11 septembre 1714. 
La haute justice [au criminel] conférait un certain prestige au seigneur et aux officiers, même si elle ne touchait qu'une partie infime de la population. De plus, les cours seigneuriales s'impliquaient dans la vie de leurs justiciables surtout en vertu de leurs compétences civiles. Elles devaient régler tous les conflits qui leur étaient adressés en matière civile. En outre le procureur fiscal agissait comme le protecteur des enfants mineurs et des orphelins et ainsi pouvait procéder dans les affaires d'héritages, le choix de tuteurs, les clôtures des inventaires de biens après décès et les partages des biens. Ce même officier travaillait aussi comme agent du seigneur; il veillait au maintien des droits féodaux et poursuivait les censitaires pour les arrérages de cens et rentes. Au service du censitaire et du seigneur, la justice seigneuriale touchait finalement un grand nombre d'habitants soumis à sa juridiction.

\section{Le fonctionnement de la justice seigneuriale de Notre-Dame-des-Anges}

Les séances de la justice seigneuriale se tenaient tout probablement dans le deuxième couvent des Jésuites construit en 1632 et situé sur la rive gauche de la rivière SaintCharles en face du petit passage appelé le "pont des sauvages" au XVIIe siècle ${ }^{37}$. Il y eut cependant quelques exceptions : ainsi le 15 décembre 1757, la séance eut lieu à Québec, le passage étant impraticable à cause des glaces ${ }^{38}$, et le 22 mars 1741 , lors d'une audience spéciale pour Sillery et Saint-Gabriel qui eut lieu à Sainte-Foy ${ }^{39}$. En outre, durant la période d'exercice des deux juges Jacques Pinguet, père et fils (1720-1748), plusieurs actes de tutelle furent dressés à leur hôtel. A moins de circonstances incontrôlables ou parfois à cause de la nature des actes, la grande majorité des audiences de la justice seigneuriale se tenaient dans la salle assignée par les seigneurs à cet effet.

Joseph-Edmond Roy affirme que la justice de Notre-Damedes-Anges tenait ses audiences le jeudi de chaque semaine et que les intendants exigeaient une séance à tous les quinze jours ${ }^{40}$.

37 J.-Edmond Roy, La justice seigneuriale, op. cit., 7; Reine Malouin, La Seigneurie de Notre-Dame-des-Anges (Québec, La Société Historique de Québec, 1955), Cahier d'histoire, no 7: 12-13. Ce site est devenu aujourd'hui l'emplacement du parc Cartier-Brébeuf.

38 CPJN, folio 3182.

39 CPJN, folio 2980.

40 J.-Edmond Roy, La justice seigneuriale, op. cit., 8. 
Un examen des registres des années de 1684 à 1688 et de 1693 à 1698 donne des résultats plus précis à ce sujet ${ }^{41}$. Dans plus de soixante pour cent des cas, l'intervalle entre deux séances fut de quinze jours, et de trois semaines dans près de vingt-cinq pour cent des cas. L'écart entre les séances fut d'une semaine dans moins de dix pour cent des cas. Donc les audiences eurent lieu normalement à toutes les deux ou trois semaines à l'exception des causes extraordinaires qui nécessitèrent, de temps à autre, l'attention immédiate de la cour.

Les vacances de la justice seigneuriale, aussi appelées "vacations" ${ }^{42}$, étaient les mêmes que celles de la Prévôté, c'est-àdire environ six semaines au temps des semences (mai-juin) et des récoltes (septembre-octobre), en plus de deux semaines à Noël et à Pâques ${ }^{43}$. Au XVIIe siècle, il y eut aussi des vacances lors de l'arrivée du vaisseau du roi. Les dates variaient d'année en année et étaient tout probablement déterminées par la température.

Une action portée devant la cour devait franchir plusieurs étapes. D'abord on s'adressait au greffier ou à un huissier pour porter une requête ou une plainte à l'attention du juge. Celui-ci répondait par une ordonnance aux parties de comparaître en cour. Un huissier communiquait cette ordonnance aux plaideurs par exploit et indiquait la date de l'audience. Le cas échéant, il assignait aussi des témoins à comparaître. Devant le juge, les personnes impliquées pouvaient plaider elles-mêmes ou se faire représenter par un procureur ${ }^{44}$. Si l'une des personnes

${ }^{41} C P J N$, folios 2760 à 2765 et 2768 à 2775.

42 Pendant l'Ancien régime, le terme "vacation" avait trois sens: un travail accompli par les juges, les frais pour ce travail et les vacances de la cour. Nous l'avons rencontré dans le sens de vacances à Notre-Damedes-Anges (CPJN, folio 2795 le 3 septembre 1704) et à la Prévôté (Archives nationales du Québec, Registres de la Prévôté de Québec (vol. 56) le 3 septembre 1720). Delalande l'emploie aussi dans ce sens. Jean Delalande, Le Conseil souverain de la Nouvelle-France (Québec, Ls-A. Proulx, Imprimeur du Roi, 1927), 142.

43 Roy affirme que les vacances étaient les mêmes que celles de la Prévôté mais ne spécifie pas la durée. J.-Edmond Roy, La justice seigneuriale, op. cit.: 8. P.-B. Mignault nous apprend que les vacances de la Prévôté duraient six semaines à la récolte et deux semaines à Pâques, mais ne mentionne rien sur l'époque des semences. P.-B. Mignault, "L'administration de la justice sous le Régime français", Revue Canadienne (1879) XVI: 110. L'examen des registres de la Prévôté et des séries de NotreDame-des-Anges révèle que Roy a bien raison et que la justice seigneuriale suit exactement les coutumes observées à la Prévôté.

44 Environ $22 \%$ des parties se faisaient représenter par un procureur au XVIIe siècle, et $30 \%$ au XVIIIe. Les procureurs étaient le plus souvent des huissiers ou des notaires. 
convoquées ne se présentait pas, un défaut était prononcé contre elle et les parties devaient se représenter à la séance suivante. Aȳant entendu les plaidoyers et examiné les pièces, le juge consultait le procureur fiscal avant de prononcer la sentence définitive. Le greffier dressait ensuite une "grosse" de la sentence qui était signifiée aux parties par un huissier. Chaque étape comportait des frais qui devaient normalement être payés par l'individu qui perdait le procès.

Le nombre de causes et leur importance varièrent énormément d'année en année. Les registres ne sont complets pour aucune année; cependant certaines séries permettent de déceler la cadence de travail de la cour seigneuriale ${ }^{45}$. Les procèsverbaux des années 1684 à $1688^{46}, 1721^{47}$, $1725^{48}, 1754^{49}$, $1755^{50}, 1757^{51}$ et $1758^{52}$, furent utilisés pour les fins de cette analyse. Le nombre de causes ${ }^{53}$ par année augmenta légèrement entre le début et la fin de la période étudiée, soit de 25 à 30 à environ $35^{54}$. Le rythme de travail à la cour seigneuriale connut des variations très marquées.

${ }^{45}$ Le manque d'organisation dans les registres rend une telle compilation très difficile puisque certains documents se trouvent écartés des registres. Seules les années où l'on trouve des cahiers complets nous assurent une bonne chance de retrouver les procès-verbaux des audiences bien que certains ne soient que partiels. Les années choisies furent celles qui représentaient le plus de certitude: couvrant le début, le milieu et la fin de la période étudiée.

$46 C P J N$, folios 2760 à 2765. Il semble y avoir des lacunes pendant l'été et au mois de décembre 1684, aux mois de janvier et décembre 1686, pendant l'été et au mois de décembre 1687, aux mois de janvier et février 1688. L’année 1685 paraît être complète.

$47 C P J N$, folios 2865 à 2866. Il manque les procès-verbaux pour janvier, juin, la première moitié de juillet et de décembre de cette année. $48 C P J N$, folios 2874 et 2876 . Il manque les mois de février et mars ainsi que toute la fin de l'année après l'audience du 5 juillet 1725 .

$49 C P J N$, folios 3102 à 3105 . Pour cette année les six premiers mois manquent.

$5_{50} C P J N$, folio 3104. Seulement deux séances se trouvent après le mois de juillet, une pour novembre et l'autre au mois de décembre.

51 CPJN, folios 3176 à 3179 et 3182 . Les séances des mois de mars et d'avril ainsi que d'août à novembre ne s'y trouvent pas.

$52 C P J N$, folios 3194 et 3195 . Il nous manque les procès-verbaux pour la fin de février et mars ainsi que la dernière séance en décembre.

53 Le rapport entre le nombre de procès-verbaux par année et le nombre de causes est très difficile à déterminer. Pour l'année 1755 il y avait 39 procès-verbaux pour 34 causes; cependant pour la plupart des années il n'y a qu'un procès-verbal pour chaque cause.

54 Pour les années les plus complètes au XVIIe siècie, soit 1685 et 1688 , le nombre de procès-verbaux est de 34 et 26 respectivement. Pour celles du XVIIIe, soit 1755 et 1758 , ils sont au nombre de 39 à chaque année. 
TABLEAU I

Nombre de procès-verbaux par mois 55

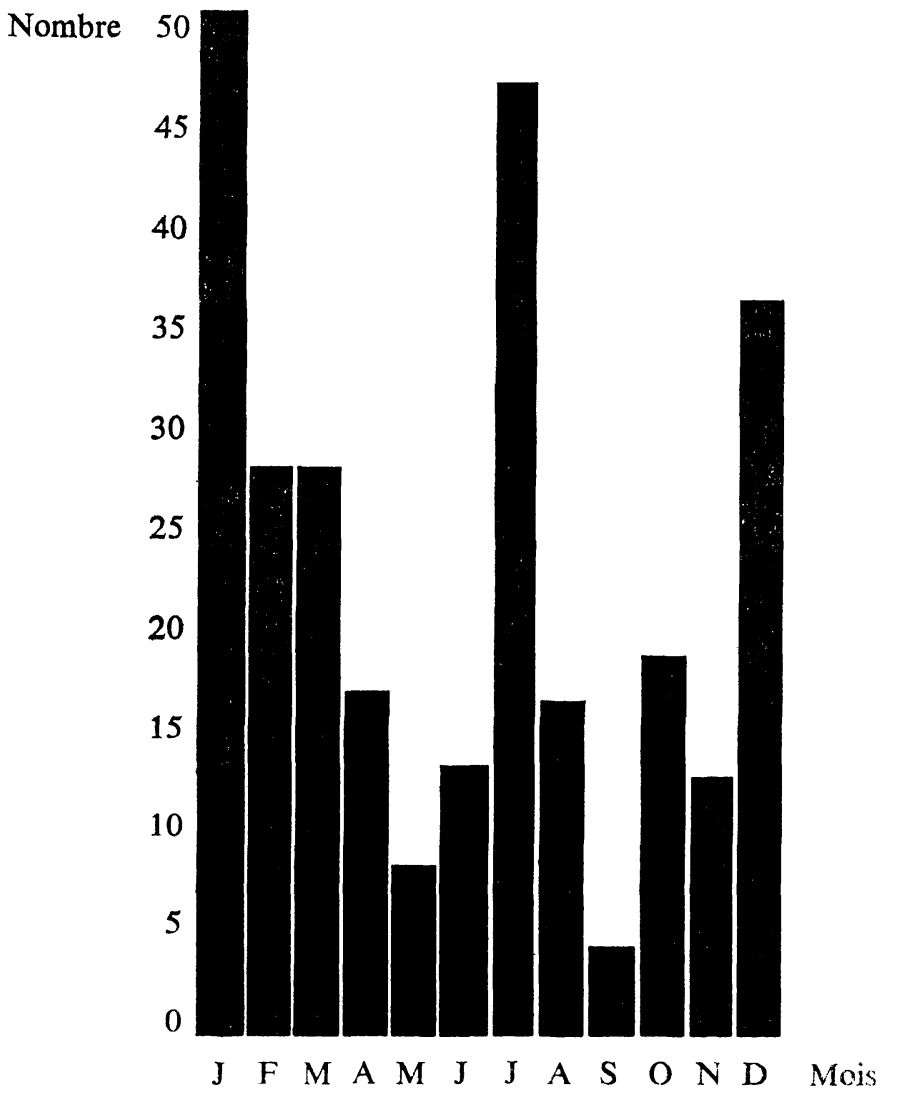

Les deux tiers de l'activité de cette justice eurent lieu entre décembre et mars et en juillet: mois pendant lesquels les besognes des habitants étaient plus légères.

Les causes portées devant la justice seigneuriale étaient assez variées.

${ }^{55}$ Ce tableau est basé sur les dix années citées ci-haut. 
TABLEAU II

Genre de causes 56

Genre

Commerce

XVIIe

XVIIIe

Héritage

Propriété

Droits seigneuriaux

Divers

Inconnus
$32(66,6)$

$23(27,7)$

$17(20,5)$

$6(12,5)$

$17(20,5)$

$5(10,4)$

$7(8,4)$

$5(10,4)$

$4(4,8)$

$15(18,0)$

Les actions de type commercial (la récupération de dettes, l'échange ou vente de marchandises, les réclamations pour des gages ou des services non-fournis) dominèrent l'activité de la cour au XVIIe siècle. Au XVIIIe siècle, ce genre de contestation tint toujours la première place bien que son importance relative ait diminué. Les procès concernant les lignes ou titres de propriété furent assez fréquents pendant toute la période, accusant une légère augmentation vers la fin du régime français. Environ dix pour cent des poursuites concernaient les droits seigneuriaux (surtout des réclamations pour le paiement d'arrérages de cens et rentes ou de lods et ventes). Les actions au sujet d'héritages étaient absentes au XVIIe siècle, mais occupaient une place importante pendant les années 1750 . Les causes diverses (petites chicanes, actions de police) furent peu nombreuses. Quinze défauts à comparaître ne donnaient aucune indication précise sur la nature du litige. La justice seigneuriale fut principalement une cour de créances. Les montants impliqués étaient assez importants pour des habitants. Au XVIIe siècle, ceux-ci se disputaient pour des sommes variant entre 10 et 25 livres dans près de la moitié des actions de type commercial. Dans quatre procès, les comptes se chiffraient entre 124 et 260 livres. Les poursuites pour des droits seigneuriaux portaient sur des montants variant entre 24 et 143 livres. Au XVIIIe siècle, les sommes réclamées dans les procès de type commercial allaient de 24 à 50 livres dans la moitié des cas. Les seigneurs poursuivaient des censitaires pour des arrérages de cens et rentes s'élevant de 23 à 185 livres.

56 Ce tableau provient d'une analyse des causes des années 1684 et 1685 pour le XVIIe siècle et 1754,1755 et 1758 pour le XVIIIe. Les pourcentages sont entre parenthèses. 
La grande majorité des causes jugées étaient de nature civile. Cependant seize procédures criminelles ont été conservées dans les registres; près de la moitié de celles-ci furent instruites par le juge Guillaume Roger entre 1685 et $1700^{57}$. Pierre Haimard en jugea trois ${ }^{58}$. Jacques Pinguet (père) eut à se prononcer une fois en $1724{ }^{59}$. Enfin le juge Paul-Antoine-François Lanoullier fit cinq instructions de ce genre entre 1754 et $1758^{60}$. En plus de régler la plupart des affaires civiles intéressant ses justiciables, la justice seigneuriale pouvait aussi agir, si nécescaire, pour punir tous les délits commis dans sa juridiction.

Outre les procès proprement dit, la justice seigneuriale était apte à se prononcer dans les affaires d'une famille au décès d'un des conjoints. Le juge procédait alors à l'apposé des scellés sur les biens du défunt en attendant que l'inventaire soit fait par un notaire. Ensuite s'il y avait des enfants mineurs, une assemblée de parents et amis devait être convoquée pour élire un tuteur et un "subrogé tuteur". Au préalable, les parents soumettaient une requête pour la tenue d'une assemblée à la cour; cette dernière y consentait toujours mais se réservait le droit de dresser l'acte de tutelle. Une fois l'inventaire terminé, le juge procédait à sa clôture et fixait l'état de la communauté à sa dissolution. Chaque fois que le tuteur voulait passer un contrat concernant les biens des mineurs sous sa charge, il devait présenter une requête pour assembler les parents et amis. A la suite de cette assemblée, on lui octroyait un acte d'autorisation pour passer le contrat. Le tuteur pouvait aussi recourir aux services de la cour pour procéder à une vente ou à un bail judiciaire. Enfin, lorsque l'enfant mineur atteignait sa majorité, il pouvait exiger un compte rendu de la gérance de ses biens par son tuteur et même le poursuivre dans les cas de gaspillage.

Les actes de ce genre ne commencèrent à prendre vraiment de l'importance dans les registres qu'au début du XVIIIe siècle. Avec l'accroissement de la population, il paraît tout à fait

$57 C P J N$, folio 2760 , le 7 juillet 1705 ; folio 2769 , le 6 septembre 1696 , les 8 et 11 novembre 1697, le 22 septembre 1698 ; folio 2777 , les 14 et 25 novembre 1699; folio 2779, le 4 mars 1700 ; Anonyme, "La haute justice", loc. cit., 187-190, le 14 septembre 1695 .

$58 C P J N$, folio 2796 , le 7 juillet 1705 ; folio 2800 , les $24,27,28$ et 30 juillet, les $2,6,11$ et 16 août, le 22 novembre 1706 et le 21 mars 1707; folio 2813, le 1er février 1708 .

59 CPJN, folio 2875 , le 20 janvier 1724.

60 CPJN, folio 3104 , le 10 décembre 1754 , le 20 février 1755 , le 20 mars 1755 ; folio 3195 , le 5 août 1758 ; folio 3207 , les $6,7,8,9,10$ et 11 novembre 1758. 
normal de trouver plus d'actes concernant les affaires familiales. Le petit nombre de ces actes au XVIIe siècle peut aussi s'expliquer du fait que ceux-ci figuraient habituellement en annexes détachées des registres d'audience et qu'ils furent ainsi plus sujets à être perdus. Il ne faut pas non plus écarter la possibilité que les gens eurent recours à la Prévôté pour dresser ces documents. Cependant les habitants semblent avoir reconnu la primauté du juge local en cette matière ${ }^{61}$.

Il est difficile de déterminer quelle proportion du travail des officiers la confection des actes administratifs constituait puisque le nombre de ces documents variait énormément d'année en année. L'analyse de la période 1754-1758 fournit quelques indices à ce sujet.

\section{TABLEAU III}

Nombre de pièces par année concernant tutelles et inventaires (1754-1758)

$\begin{array}{lcccc}\text { Année } & \begin{array}{c}\text { Actes de } \\ \text { tutelle }\end{array} & \begin{array}{c}\text { Requêtes } \\ \text { d'assemblée }\end{array} & \begin{array}{c}\text { Actes } \\ \text { d'autorisation }\end{array} & \begin{array}{c}\text { Clôture } \\ \text { d'inventaire }\end{array} \\ 1754 & 8 & 10 & 1 & 162 \\ 1755 & 4 & 6 & 2 & 263 \\ 1756 & 7 & 8 & 0 & 364 \\ 1757 & 3 & 5 & 0 & 265 \\ 1758 & 10 & 5 & 0 & 266\end{array}$

61 En 1750 un tuteur qui voulut se démettre de sa charge, demanda à Lanoullier d'annuler l'élection qui avait eu lieu devant le lieutenant général de la Prévôté, celui-ci n'étant pas son "juge naturel". $C P J N$, folio 3034, le 22 mai 1750. Entre 1750 et 1754 seulement trois clôtures d'inventaire dressées par la Prévôté concernaient des habitants des paroisses de la juridiction de Notre-Dame-des-Anges. Dans deux cas les individus venaient de Lorette et auraient pu demeurer dans la seigneurie de Maure. Archives nationales de Québec, Registres de l'état civil, Clôtures d'inventaire, 1744-1759. Pendant cette même période, le juge de Notre-Dame-desAnges dressait 11 clôtures d'inventaire.

$62 C P J N$, folios 3106 à 3122 .

63 CPJN, folios 3123 à 3140 .

$64 C P J N$, folios $3143,3146,3150,3152,3156,3157,3159,3161,3165$ à $3167,3169,3171,3174$ et 3175 .

$65 C P J N$, folio 3179 , le 15 mars et le 7 juillet, folios $3185,3186,3188$, 3189 et 3191 .

66 CPJN, folio 3195 , le 20 juin, les 9,13 et 20 juillet, le 25 octobre, les 24 et 27 novembre 1758 ; folios 3196 à $3200,3204,3205,3209,3210,3214$ et 3216 . 
Ce tableau ne donne qu'une idée imparfaite du nombre de pièces puisqu'il manque plusieurs requêtes ou actes dans les registres. Ainsi, en 1758, au moins dix requêtes d'assemblée devraient s'y trouver, mais il n'en existe que cinq et, de ce nombre, une ne réfère à aucun des actes de tutelle conservés. Ce travail, bien que beaucoup moins accaparant que le jugement des litiges (39 procès contre 10 actes de tutelle et 2 clôtures d'inventaire en 1758), démontre le rôle que joua la justice seigneuriale dans la protection des droits des mineurs.

Une autre fonction de la cour seigneuriale fut la police ${ }^{67}$. Nous avons relevé huit ordonnances de police décrétées par les juges de Notre-Dame-des-Anges. De plus, plusieurs ordonnances du Conseil souverain, de la Prévôté de Québec et des intendants furent enregistrées et publiées dans cette juridiction. Le procureur fiscal était chargé de veiller à l'exécution de ces règlements et de traduire les contrevenants en justice. Plusieurs ordonnances assuraient l'application locale de règles générales édictées par des instances supérieures: la fixation du prix du pain ${ }^{68}$, la détermination des poids et mesures à être emploýés par les cabaretiers ${ }^{69}$, l'obligation de faire garder les bestiaux pendant la période des semences ${ }^{70}$ et l'érection de clôtures autour des prairies ${ }^{71}$. D'autres ordonnances visaient à corriger des situations particulières à l'intérieur de la juridiction, tels les règlements concernant la réparation ou la construction des chemins ${ }^{72}$, interdisant le pâturage des bestiaux de la ville sur la commune même s'ils étaient gardés par des habitants de la seigneurie ${ }^{73}$, défendant aux chasseurs de traverser les champs ${ }^{74}$

${ }^{67}$ Seul Eccles accorde cette compétence à la justice seigneuriale, pourtant elle existait définitivement. Eccles, op. cit., 73, note 24 .

$68 C P J N$, folio 2872, Enregistrement d'une ordonnance du Conseil souverain le 7 avril 1686.

69 CPJN, folio 3153, Ordonnance de Paul-Antoine-François Lanoullier du 4 décembre 1756.

70 CPJN, folio 2799, Ordonnance de Pierre Haimard du 22 mai 1705. Une ordonnance au même effet se répétait à la Prévôté tous les ans entre 1721 et 1728.

$71 C P J N$, folio 2756, Ordonnance de Pierre Duquet du 18 juillet 1679 et l'enregistrement d'une ordonnance du Conseil souverain le $1 \mathrm{er}$ avril 1686, folio 2763 .

72 CPJN, folio 2762, Ordonnance de Guillaume Roger du 30 mai 1686 et folio 2796, de Pierre Haimard du 16 juillet 1705 .

73 CPJN, folio 3012, Ordonnance de Paul-Antoine-François Lanoullier du 19 mars 1750.

74 CPJN, folio 3154, Ordonnance de Paul-Antoine-François Lanoullier du 8 mai 1756. 
ou fixant le prix du passage de la rivière Saint-Charles ${ }^{75}$. La police, en plus d'assurer le respect de l'ordre et de corriger des abus, pouvait constituer un mécanisme d'administration pour les seigneurs et protéger leurs droits féodaux.

Les seigneurs profitaient dans une certaine mesure de l'établissement d'une justice sur leurs fiefs. Les Jésuites se servirent aussi de leur tribunal pour réclamer les arrérages de cens et rentes et le paiement de lods et ventes. Il y eut d'autres poursuites pour réunir des terres inhabitées au domaine ${ }^{76}$, pour exiger des contrats de concessions 77 et pour affermer leurs droits seigneuriaux ${ }^{78}$. La cour locale offrait donc un moyen de régler des problèmes d'administration générale.

Si la justice seigneuriale pouvait servir aux seigneurs, elle offrait aussi plusieurs avantages aux censitaires. L'établissement d'un tel tribunal évitait à l'habitant un voyage au siège de la justice royale. Dans le cas de Notre-Dame-des-Anges un tel déplacement n'aurait pas été très onéreux, mais dans les seigneuries plus lointaines, telles celles de Beaupré, de l'Ile a'Orléans et de la Rivière du Sud, la justice seigneuriale devait être fort appréciée. De plus les frais à la cour subalterne n'étaient que les deux tiers de ceux de la juridiction royale et les notaires seigneuriaux ne pouvaient exiger que la moitié du tarif des notaires royaux.

\section{Les frais de justice}

Le juriste français du XVIIe siècle, Charles Loyseau, qualifia les justices seigneuriales de "mangeries de villages" 79, opinion qui était partagée au Canada par certains comme Ruette d'Auteuil qui décrivit la justice de Sillerỹ comme une charge "des plus onéreuses [...] sans nécessité" ${ }^{80}$. Mais sans doute

${ }^{75} C P J N$, folio 2864, Ordonnance de Jacques Pinguet (père) du 23 mars 1720

$76 C P J N$, folio 2769 , les 12 mars et 9 juillet 1693.

$77 C P J N$, folio 2803 , le 4 avril 1706.

78 CPJN, folio 2769 , le 7 février 1697. Il y eut 3 causes concernant le droit de mouture à cette date.

79 Combier, op. cit., $\mathrm{x}$.

80 Le procureur général critique surtout Sillery puisque la distance de Québec n'est pas assez considérable pour justifier un siège particulier. Il adresse cependant des critiques plus générales quand il se plaint qu'au Canada "on ne paye pas la tournée des plaideurs", c'est-à-dire on ne rembourse pas les parties pour le temps perdu; ce qui devait être une charge assez accablante. Ruette d'Auteuil, loc. cit., 20-21 et 30-31. A NotreDame-des-Anges la cour attribuait des salaires aux témoins mais non aux parties en cause. 
faut-il préciser que ces deux juristes désiraient que le nombre de niveaux de justice fût réduit au profit des officiers royaux. Les critiques blâmaient souvent la rapacité des officiers qui saignaient les justiciables pour se procurer des revenus convenables. La condition matérielle des officiers fut donc en cause.

On a souvent mentionné la misère des officiers de justice sous le régime français ${ }^{81}$; cependant les titulaires de fonctions de Notre-Dame-des-Anges ne furent pas réduits à la pauvreté et quelques-uns même connurent une certaine prospérité ${ }^{82}$. L'évaluation des revenus de ces hommes s'avère presque impossible vu le cumul de fonctions. Un poste dans la justice de NotreDame-des-Anges ne représentait jamais la seule occupation des titulaires; aux niveaux supérieurs, ils semblent avoir recherché le prestige tandis que les greffiers et huissiers y trouvaient un revenu d'appoint ${ }^{83}$. Les officiers pouvaient assurer leur subsistance sans cet emploi et l'on n'a pas de preuve de cupidité de leur part.

L'ordonnance d'Orléans de 1560 décréta que les seigneurs devaient assurer des "gages honnêtes" à tous leurs officiers, mais rien n'indique que cette ordonnance fut respectée en France ${ }^{84}$. Les Jésuites de Nouvelle-France devaient payer leurs officiers, mais le montant exact des traitements demeure inconnu. Jean Laurent dit Lortie, procureur fiscal, reçut 30 livres par année entre 1740 et 1744 avec une rétribution additionnelle de 6 livres en 1742 et 1744 pour avoir fait le recensement ${ }^{85}$. Ce montant constitue le dixième de la rémunération du Procureur du

81 André Vachon est celui qui a le plus répandu cette idée par les nombreuses notices biographiques qu'il a rédigées sur ces fonctionnaires. Il cite Jean-Etienne Dubreuil, cordonnier, huissier royal, notaire, procureur fiscal et ensuite juge de Notre-Dame-des-Anges comme un exemple type de la "misère des officiers de justice" (Dictionnaire biographique du Canada, II: 209). A son décès Dubreuil était effectivement assez pauvre n'ayant que 204 livres de biens, mais sept années d'infirmité devaient avoir sérieusement affecté sa fortune. Un inventaire qui fixait l'état de sa première communauté en 1705 , précisait qu'il possédait des biens meubles d'une valeur de 3.786 livres plus une maison. En 1715 lors de son troisième mariage sa fortune montait à 2.550 livres de biens meubles, sa maison, un emplacement et un verger de 60 arbres dont 30 "portaient fruits". Dickinson, op. cit., 86-87.

82 Dickinson, op. cit., 82-89, 99-102, 113-115 et 123-127.

83 Ibid., 71-82, 94-99, 108-113 et 119-123.

84 Giffard, op. cit., 87-88; et Combier, op. cit., 6.

85 Archives nationales du Québec, Biens des Jésuites (Cote QBC 18), 1: 108. André Vachon affirme que les seigneurs payèrent les officiers, mais ne mentionne pas les montants versés. Vachon, loc. cit.: xxiii. 
roi à la Prévôté ${ }^{86}$ et si les mêmes proportions avaient été gardées pour les autres officiers, le juge aurait dû recevoir 70 livres et le greffier, 10 livres. Ces chiffres, bien que discutables, semblent vraisemblables. Il se peut aussi que seul le procureur fiscal, l'officier le plus directement attaché au service du seigneur, fut rémunéré, ce qui diminuait de beaucoup les frais des seigneurs.

Les gages des officiers ne formaient qu'une partie de leur revenu global; les émoluments composaient la portion la plus substantielle ${ }^{87}$. Ceux-ci furent déterminés par le Roi dans le "tarif" de 1678 et ensuite revisés en $1749{ }^{88}$. Cette ordonnance fixait la rémunération des officiers des cours subalternes aux deux tiers de ceux de la cour royale. Il est difficile d'établir si ces tarifs étaient scrupuleusement respectés par la justice de Notre-Dame-des-Anges, mais quelques indices laissent croire que les officiers augmentèrent très légèrement les taux ${ }^{89}$. Nous avons pu relever 75 causes pour lesquelles des dépens sont indiqués dont 47 à la sentence définitive et 28 avant la fin du procès. Ces données sont trop maigres pour arriver à des conclusions définitives, mais elles donnent une idée des frais que pouvaient encourir les parties lors d'un procès.

Une cause sans complications, dont l'issue pouvait être déterminée lors de la première comparution en cour, coûtait de une à six livres au condamné. Tel fut le cas dans un peu plus du tiers des procès observés. Si l'altercation nécessitait deux présences en cour ou l'examen de documents, les frais montaient assez rapidement et se situaient entre 6 et 12 livres. Les causes plus compliquées, souvent des disputes sur les lignes de propriété ou des réclamations pour dommages causés par des

86 Cameron Nish, Les bourgeois-gentilshommes de la Nouvelle-France (1729-1748) (Montréal, Fides, 1968), 146.

87 Roland Mousnier, La vénalité des offices sous Henri IV et Louis $X I I I$ (Rouen, Editions Maugard, s.d.), 55-56.

88 Québec, op. cit., I: 99-102 et 609-612. Cette ordonnance fut enregistrée à Notre-Dame-des-Anges seulement le 11 octobre 1685 . CPJN, folio 2760. Malgré ces règlements, Gérard Filteau a pu affirmer que tous les frais étaient abolis dans ces cours! Gérard Filteau, La naissance d'une nation, Tableau du Canada en 1755 (Montréal, Editions de l'Action canadienne-française, 1937), I: 106-107.

89 En 1705 le juge Haimard s'attribua 2 livres, 2 sols, 6 deniers "de france" pour une vacation; tandis qu'il aurait dû ne charger que 2 livres, 1 sol, 10 deniers du pays, $C P J N$, folio 2797 , le 17 décembre 1705 . Le greffier Geneste réclama 18 livres pour son transport en campagne pendant trois jours, soit presque le taux du greffier de la Prévôté, CPJN, folio 3065 , les 14, 15 et 16 avril 1751. Les taux des assignations des huissiers nous semblèrent aussi quelque peu élevés pour se conformer à ceux spécifiés, mais nous ne disposions que de peu d'exemples. 


\section{TABLEAU IV}

Les frais de justice
Nombre
de causes
pendant le
procès
à la sentence
définitive

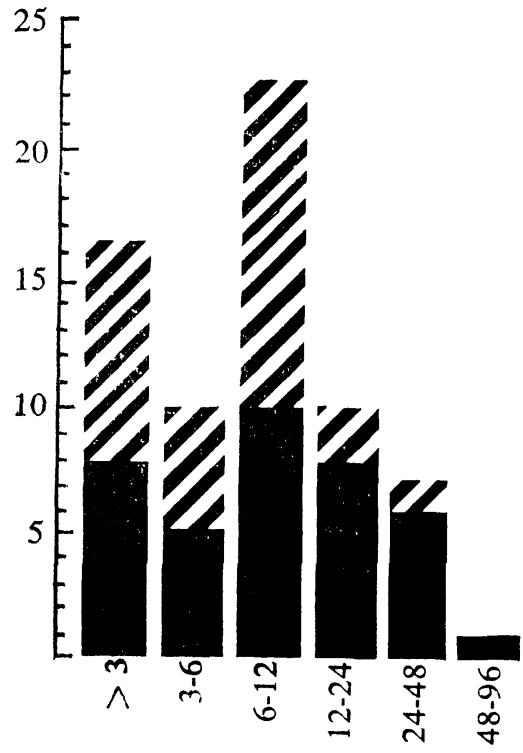

Coût en livres

bestiaux qui exigeaient le recours à des experts (arpenteurs ou évaluateurs), coûtaient entre 12 et 24 livres. Trois quarts des actions dont les dépens montaient entre 24 et 28 livres étaient de nature criminelle. Dans une seule cause les dépens furent supérieurs à 50 livres. Celle-ci nécessita l'arpentage des terres et coûta 96 livres. Près de la moitié des actions entreprises devant la cour seigneuriale furent "taxées" entre 6 et 24 livres et environ 45 pour cent de celles-ci entraînèrent des frais de 10 à 14 livres ${ }^{90}$.

90 Puisque nous ne pouvions savoir le coût final du procès quand les dépens ne furent indiqués qu'au milieu, nous ne nous sommes pas servis de ces cas dans le calcul des pourcentages. Ces frais ne comprennent pas les amendes, mais uniquement ce que les particuliers furent obligés de débourser aux officiers et les salaires des témoins dans les procès criminels. 
Ces chiffres seraient difficiles à évaluer sans moyen de comparaison. Une étude récente d'André Frenière portant sur la série des "Taxes de dépens du Conseil supérieur", révèle le coût de la justice à cette cour. Pour les deux années analysées (1715 et 1745 ) les frais moyens pour un procès s'élevèrent entre 110 et 125 livres ${ }^{91}$. Bien que la présence de causes très coûteuses hausse cette moyenne, la moins élevée se chiffre à 51 livres et plus de cinquante pour cent occasionnèrent des dépens entre 51 et 100 livres ${ }^{92}$. Les frais à la Prévôté devaient aussi être supérieurs à ceux de la justice seigneuriale à cause du tarif.

Les coûts des divers actes passés par la juridiction pour régler les héritages et les affaires de tutelle varièrent selon l'époque. Au début de la période étudiée ils étaient “taxés" entre 36 et 60 sols. Pendant les années d'inflation entre 1715 et 1720 des actes de tutelle coûtaient jusqu'à 14 livres en monnaie de cartes ${ }^{93}$. Vers la fin du régime français, les frais étaient plus stables: un acte de tutelle ou d'autorisation exigeait des dépens de 3 livres 5 sols et une clôture d'inventaire ou une affirmation de compte coûtait 5 livres 5 sols. La rédaction d'une requête par le greffier encourait des frais entre 15 et 30 sols; plusieurs requêtes furent rédigées par les huissiers et dans l'unique cas où l'émolument fut noté, Nicolas Jacques reçut 30 sols ${ }^{94}$.

Malgré le nombre limité d'exemples, les amendes ne semblent pas avoir été exhorbitantes, la plupart étant fixées entre 2 et 8 livres. Les coupables durent débourser 3 et 5 livres dans des causes de voie de fait ${ }^{95}, 3$ et 6 livres pour calomnie ${ }^{96}$ et 2 livres pour "l'enlèvement d'une terre avec violence" 97 ! Dans des causes impliquant les droits des seigneurs, l'amende fut de 2 livres pour avoir détourné le ruisseau qui alimentait le moulin seigneurial ${ }^{98}$ et de 8 livres pour une personne qui refusa de faire moudre ses grains au moulin banal ${ }^{99}$. Dans cette dernière

91 André Frenière, "Les taxes de dépens dans le revenu des officiers de justice du Conseil supérieur: 1715 et 1745". Travail manuscrit présenté à Jacques Mathieu lors d'un séminaire de maîtrise (Québec, Université Laval, Institut d'histoire, mars 1972), 14-15.

92 Ibid., 16.

93 CPJN, folio $28621 / 2$ les 16 février et 9 mars 1719.

$94 C P J N$, folio 2869 , le 5 juillet 1728 . 1697.

95 CPJN, folio 2778 , le 25 novembre 1697 et folio 2769 , le 11 novembre 1754.

96 CPJN, folio 2769 , le 22 septembre 1698 et folio 3104 , le 19 décembre

97 CPJN, folio 2864, le 7 novembre 1720.

$98 C P J N$, folio 2876 , le 26 juin 1725 .

99 CPJN, folio 2874 , janvier 1725 . 
cause, l'inculpé mérita aussi "40 sols d'amende pour nous (le juge) avoir insulté sur notre Siège". Un autre cas d'outrage au tribunal en 1755 entraîna une amende de 6 livres ${ }^{100}$. La plus forte peine fut infligée par le juge Roger qui exigea 50 livres de ceux qui avaient aidé un meurtrier à fuir la prison ${ }^{101}$. Dans une action de police un cabaretier fut obligé de débourser 24 livres pour avoir faussé ses mesures ${ }^{102}$. Les amendes étaient rares et ne contribuaient pas à hausser le coût de la justice. Il est aussi à noter que les bénéfices ainsi obtenus étaient versés au profit de la paroisse de Charlesbourg; ni les seigneurs, ni les officiers n'en profitèrent.

En dehors des revenus provenant directement des procès et actes dressés par la justice, la possession même du greffe comportait une rémunération dont bénéficiaient le juge et le greffier. Outre les papiers de la juridiction, les études des notaires seigneuriaux étaient déposées au greffe, et la recherche pour un titre de concession ou un acte quelconque occasionnait des frais. Si on peut juger de l'importance de ces revenus par l'acharnement avec laquelle le seigneur Duchesnay essaya de conserver les archives de Notre-Dame-des-Anges, les bénéfices ne devaient pas être négligeables ${ }^{103}$. Il serait surprenant de voir un seigneur défendre les droits de son greffe avec autant de violence si lui aussi n'en retirait aucun profit. Ces archives furent tout probablement mises à ferme comme à Montréal ${ }^{104}$.

En comparaison avec ceux de la justice royale, les frais encourus dans une cour seigneuriale n'étaient pas élevés. Par ce fait, la juridiction locale offrait aux habitants une alternative à la Prévôté, ce qui explique son activité soutenue. Néanmoins, il reste que les dépens exigés représentaient des sommes importantes pour les justiciables. Même les petites causes, les actes de tutelle et les clôtures d'inventaire équivalaient à presque deux semaines de revenus pour un habitant et deux à trois jours de travail pour un ouvrier spécialisé ${ }^{105}$. Les frais de justice étaient donc considérables.

$100 C P J N$, folio 3104 , le 30 novembre 1755.

101 Anonyme, "La haute justice", loc. cit.: 190.

$102 C P J N$, folio 3104 , le 20 février 1755.

103 Le juge Lanoullier accusa Duchesnay de s'être approprié des archives "apparemment pour un vil intérêt pour augmenter le revenu de son greffe".J.-Edmond Roy, op. cit., 22.

104 Louise Dechêne, "L'évolution du régime seigneurial au Canada. Le cas de Montréal aux XVIIe et XVIIIe siècles", Recherches sociographiques (1971), XII : 163. 41-42.

105 Ces calculs sont basés sur les chiffres donnés par Nish, op. cit., 


\section{Conclusion}

La justice seigneuriale, négligée par tant d'historiens, joua cependant un rôle important dans la vie de ses justiciables. Outre le règlement des disputes entre ses censitaires, la juridiction jugeait parfois des causes très sérieuses; le droit à la haute justice ne demeurait pas un titre inopérant et plusieurs causes criminelles furent instruites au cours de la période étudiée. Les activités de cette cour subalterne ne se limitaient donc pas seulement aux "petites chicanes". Ses fonctions dans le domaine des règlements des héritages et de la protection des droits des mineurs lui accordaient certainement une grande importance auprès de ceux qui y étaient soumis. Dans une société où la famille formait la base du système légal, la responsabilité de veiller à ce que les rapports entre les membres soient conformes à la Coutume était très lourde. Les justices seigneuriales constituaient donc des mécanismes utiles de l'administration judiciaire de la Nouvelle-France.

Notre-Dame-des-Anges ne fut pas la seule justice seigneuriale qui ait opéré d'une manière continue pendant le régime français. Les seigneuries de Beauport, de Beaupré et de l'Ile d'Orléans avaient des cours locales depuis les années 1660, et la seigneurie de la Rivière du Sud, depuis $1736^{106}$. Si l'on peut juger d'après les causes portées en appel devant la Prévôté de Québec, ces juridictions étaient aussi actives que Notre-Damedes-Anges. Harris affirme que le petit nombre de procès de ce genre devant les instances supérieures indique un manque d'activité au niveau seigneurial ${ }^{107}$. Ses conclusions sont tout à fait gratuites et sans aucun fondement. Entre 1715 et 1720 inclusivement, seulement cinq causes de Notre-Dame-des-Anges furent portées en appel devant la Prévôté ${ }^{108}$ mais les registres de cette cour seigneuriale démontrent qu'elle fonctionnait norma-

106 Dans la $C P J N$, nous retrouvons aussi plusieurs commissions d'officiers pour des endroits tel que Grande Anse-La Bouteillerie (folio 2018), Lauzon (folio 2020 et 2089), Bellechasse (folio 2042) Champlain (folio 2050 1/2), Cap de la Magdeleine (2050 3/4) Batiscan (folios $20531 / 2$, 2068 et $20891 / 2$ ), Yamaska-Saint-François (folios 2073 1/2 et 2076) ainsi que Saint-Vallier (2077 et 2078).

107 Harris, op. cit., 190.

108 Archives nationales du Québec, Registres de la Prévôté de Québec (vol. 52), le 15 décembre 1716; les 20 avril et 2 août 1717; (vol. 53), le 29 juillet 1718 ; le 2 décembre 1718 , les 10 et 31 janvier 1719 ; le 17 janvier et 7 février 1719. Une sentence seigneuriale fut maintenue, deux furent renversées et la sentence définitive dans deux autres cas est inconnue. 
lement pendant ces années. Deux autres explications semblent plus réalistes: soit que la qualité de la justice laissait peu à désirer, soit que les frais de la Prévôté aient diminué l'ardeur des contestataires.

Dans le gouvernement de Québec, les seigneuries les mieux établies avaient des cours seigneuriales vers la fin du régime français. Si elles ne représentaient qu'un petit pourcentage de la totalité des fiefs concédés dans ce gouvernement, elles contenaient cependant près de $60 \%$ de la population. Dans le gouvernement de Trois-Rivières, les seigneuries les plus considérables avaient leurs propres tribunaux. A Montréal, seule exista la justice seigneuriale jusqu'en $1693^{109}$. A la suite de la création d'une cour royale à Montréal, la justice subalterne ne disparut pas complètement; les Sulpiciens conservèrent la basse justice sur toute l'île pour les cens et rentes et autres redevances seigneuriales avec la faculté de haute justice dans l'enclos du Séminaire et sur la ferme Saint-Gabriel ${ }^{110}$. Cette juridiction devait être assez active puisque le bail pour le greffe avait été fixé à 200 livres pendant le premier quart du XVIIIe siècle ${ }^{111}$. Les recettes provenant du greffe devaient ainsi couvrir toutes les dépenses qu'entraînait le maintien de la cour et même procurer un petit revenu supplémentaire aux seigneurs.

La disparition des documents a souvent, à tort, convaincu les historiens que ces juridictions n'ont jamais existé. Cependant, les archives et greffes étaient conservés dans les maisons des greffiers et il n'est point surprenant que beaucoup de documents aient disparu lors d'incendies ou de déménagements. Des justices seigneuriales existaient et fonctionnaient normalement en offrant aux censitaires une alternative aux cours royales, parfois lointaines, et toujours plus dispendieuses. Toute analyse du régime seigneurial en Nouvelle-France devrait tenir compte de ce facteur en évaluant l'importance de la seigneurie et les mécanismes administratifs dont disposait le seigneur.

109 Robert Lahaise, Le bailliage montréalais et ses officiers de justice. Thèse pour l'obtention d'un Diplôme d'Etudes supérieures, Institut d'histoire (Québec, Université Laval, 1968), xxi et 147 p.

110 Pierre-Georges Roy, "Correspondance de Vaudreuil", loc. cit., Mémoire du Roi à MM. de Vaudreuil et de Beauharnois du 14 juin 1704, 37. 111 Dechêne, loc. cit., 163. 